\title{
Lean muscle mass and bone mineral content are predictors of lung function in ex-smokers with or without COPD
}

\author{
Joanne M Smart ${ }^{1 *}$, Vanessa M McDonald ${ }^{2,3}$, Peter G Gibson ${ }^{1,2}$, Peter AB Wark ${ }^{1,2}$ and Lisa G Wood $^{2,4}$ \\ ${ }^{1}$ School of Medicine and Public Health, University of Newcastle, Newcastle, NSW, Australia \\ ${ }^{2}$ Centre for Asthma \& Respiratory Diseases, Hunter Medical Research Institute, Australia \\ ${ }^{3}$ School of Nursing and Midwifery, University of Newcastle, Newcastle, NSW, Australia \\ ${ }^{4}$ School of Biomedical Sciences and Pharmacy, University of Newcastle, Newcastle, NSW, Australia
}

\begin{abstract}
Objectives: To examine body composition in relation to lung function, inflammation and physical activity in ex-smokers with or without Chronic Obstructive Pulmonary Disease (COPD).

Methods: One hundered and seven (107) adults, $\geq 35$ years (16 never smokers, 68 ex-smokers without COPD, 23 ex-smokers with COPD) were recruited. Body composition (dual energy $\mathrm{x}$-ray absorptiometry), spirometry, airway (sputum cell counts) inflammation, systemic inflammation (CRP, IL-6, leptin), physical activity and quality of life were assessed. Partial correlation, linear and multiple regression analyses were performed to identify predictors of lung function and associations with body composition.
\end{abstract}

Results: Lean muscle mass (LMM) and bone mineral content (BMC) were positively associated with $\mathrm{FEV}$ ( $\mathrm{r}=0.447, p<0.001$ and $\mathrm{r}=0.335, p<0.01$ respectively) and FVC $(\mathrm{r}=0.507, p<0.001$ and $\mathrm{r}=0.234, p<0.05$ respectively). Fat mass was negatively associated with FEV $(\mathrm{r}=-0.443, p<0.001)$ and $\mathrm{FVC}(\mathrm{r}=-0.518, p<$ 0.001). CRP, IL-6 and leptin were negatively associated with FEV and FVC. In multiple regression models, LMM and BMC remained significant predictors of $\mathrm{FEV}_{1}, \mathrm{FVC}$ and $\mathrm{FEV}_{1} / \mathrm{FVC}$.

Discussion: LMM and BMC are positively associated with lung function in ex-smokers. Physical activity and systemic inflammation were negatively associated with body composition. Strategies improving exercise capacity and reducing systemic inflammation may improve body composition and outcomes in ex-smokers.

\section{Introduction}

Body composition is a predictor of mortality in chronic obstructive pulmonary disease (COPD). A low body mass index (BMI) has been associated with increased mortality and morbidity, while those with a higher BMI (between 30 and $35 \mathrm{~kg} / \mathrm{m}^{2}$ ) have better health outcomes $[1,2]$. The latter being described as the 'obesity paradox'. Obesity is now a common feature of COPD, with studies reporting rates of obesity in COPD as high as $50 \%[3,4]$. The mechanism by which obesity may be protective in COPD is unknown but may involve a reduction in static lung volumes and/or the increase in fat free mass (FFM - bone, muscle and soft tissue organs) due to over-nutrition and increased weight bearing $[5,6]$.

The prevalence of osteoporosis in COPD, is higher compared to healthy controls and other chronic pulmonary diseases [7]. Both COPD and osteoporosis have common risk factors such as age and smoking. Additionally, the presence of systemic inflammation, inadequate diet, vitamin $\mathrm{D}$ deficiency, inactivity and corticosteroid use may further enhance ongoing bone loss $[8,9]$.

Both airway and systemic inflammation are involved in the pathogenesis of COPD. Airway inflammation in COPD is characterised by the presence of increased neutrophils, macrophages and lymphocytes and increased pro-inflammatory cytokines such as elastases, proteases, interleukin-8 (IL-8), leukotriene beta 4 (LTB $)$, interleukin 6 (IL-6) and tumour necrosis factor alpha (TNF- $\alpha)$ [10]. Systemically, increased levels of inflammatory markers such as $\mathrm{C}$ reactive protein (CRP), TNF- $\alpha$, IL- 6 and fibrinogen have been reported in COPD [11]. These same pro-inflammatory including leptin are expressed and released from adipose tissue [12]. It is unclear whether the increase in systemic inflammation seen in COPD is a result of overflow from the airways and/or a result of other factors such as body composition and physical activity. In COPD, lower levels of physical activity, which can lead to a loss of muscle mass, has also been associated with airflow obstruction, disease severity, higher levels of systemic inflammation, increased risk of hospital admissions, mortality and reduced quality of life $[13,14]$.

The role of inflammation and physical activity in determining body composition in ex-smokers and the association with airflow obstruction has not been comprehensively described. The aims of this study were to examine body composition, inflammation, physical activity, and lung function in healthy controls (never smokers) and ex-smokers, with or without COPD and to identify potential modifiable factors that may alter body composition and therefore outcomes in those with and who may develop COPD.

${ }^{\star}$ Correspondence to: Joanne Smart, School of Medicine and Public Health University of Newcastle, Newcastle, NSW, Australia, Tel: 612 40143947; Fax: 61 2 40143945; E-mail: Joanne.Smart@calvarymater.org.au

Key words: Body composition, ex-smokers, lung function

Received: May 27, 2019; Accepted: June 10, 2019; Published: June 13, 2019 


\section{Methods}

The study design was observational with one hundred and seven participants, aged $\geq 35$ years recruited into the study. Recruitment was in response to newspaper and radio advertising, from the John Hunter Hospital Respiratory Outpatient Clinic and Hunter Medical Research Institute Research Register between June 2009 and May 2012. 91 were ex-smokers and had ceased smoking for $>1$ year and had a $>5$ pack year smoking history. Smoking pack years were calculated using a webbased calculator [15]. 16 were healthy controls (never smokers who had never smoked or smoked $<100$ cigarettes in their lifetime). As this study primarily investigated ex-smokers with and without COPD, only 16 healthy controls were recruited. Each group was controlled for age and BMI. Exclusion criteria for all participants included: recent (past month) respiratory tract infection, current lipid lowering agents use, unstable cardiac conditions and other chronic inflammatory conditions. The study was approved by the Hunter New England and University of Newcastle Human Research Ethics Committees and participants gave written informed consent. Participants attended 2 study visits (maximum of 2 weeks between visits). Lung function testing, sputum induction, blood collection, questionnaires and body composition were completed at visit 1 . The modified shuttle walk test (MSWT) and pedometer diaries were collected and completed at visit 2. 107 participants completed visit 1, 4 participants did not complete visit 2; with one lost to follow up, one withdrew consent and two due to health issues.

\section{Lung function testing}

Pre and post bronchodilator spirometry was performed (KoKo Spirometer, Pulmonary Data Service, Instrumentation Inc., Louisville, USA) according to American Thoracic Society (ATS) recommendations. Participants were defined as having COPD if post bronchodilator forced expiratory volume in 1 second $\left(\mathrm{FEV}_{1}\right)$ /forced vital capacity $(\mathrm{FVC})$ ratio where the lower limit is 1.96 standard deviations below the mean (with a $95 \%$ confidence limit) [16].

\section{Body composition assessment}

A total body scan was performed using dual energy $\mathrm{x}$-ray absorptiometry (DEXA - Lunar Prodigy Pro Dual ${ }^{\oplus}$, GE Healthcare Madison, WI, USA version 11.3). Measures of body composition were derived from the DEXA body scan and included: total body fat mass (FM - body fat), total lean muscle mass (LMM - lean muscle and soft tissue), fat free mass (FFM - LMM and bone) and bone mineral content (BMC - bone only). Fat free mass index (FFMI) was calculated as (total $\mathrm{LMM}+$ total BMC)/height ${ }^{2}$.

\section{Airway and systemic inflammation measurement}

Online exhaled nitric oxide (eNO) was measured $\left(\mathrm{NiOX}^{\circ}\right.$ Aerocrine AB, Sweden) according to ATS guidelines. Sputum induction with hypertonic (4.5\%) saline was performed as described [17]. Lower respiratory sputum portions were selected and dispersed using dithiothreitol [17]. Sputum total cell counts and cell viability (trypan blue exclusion) were performed by haemocytometer and cytospins used for differential cell counts.

Fasting blood plasma CRP concentration was analysed by commercial enzyme-linked immunosorbent assay (ELISA - Siemens Healthcare Diagnostics Inc., Newark, DE, USA). Serum leptin (BioPlex Pro ${ }^{\mathrm{m}}$ Assay Bio-Rad Laboratories, Inc., USA) and IL-6 (R\&D systems, Minneapolis, $\mathrm{MN}$, USA) was stored at $-80^{\circ} \mathrm{C}$ and analysed by commercial ELISA.

\section{Exercise endurance and physical activity measures}

Modified Shuttle Walk Test (MSWT): The MSWT was administered according to the recommended guidelines [18]. The number of shuttles completed were recorded and multiplied by 10 ( 1 shuttle $=10$ metres). The MSWT allowed those with and without disease to run or walk according to their functional capacity. The MSWT was selected over the six-minute walk test (6MWT) due to the limitations of the 6MWT to define exercise endurance in those with better lung function [19].

Daily step count - pedometer: Single function pedometers were used to record the number of steps per day (Digi-walker SW-200, Yamax) and daily results were recorded in a diary. Pedometers were worn between visits 1 and 2 for 6 days with the average number of steps per day calculated.

International Physical Activity Questionnaire (IPAQ): The selfadministered, long version IPAQ was used to estimate physical activity, using last week recall. Walking, moderate, vigorous and total physical activity, were expressed as MET-minutes/week (metabolic equivalent) according to the IPAQ analysis guidelines [20].

St George's Respiratory Questionnaire (SGRQ): The selfadministered SGRQ was used to measure the impact of symptoms, activity and disease on daily life. Total score and activity domain were used in the analysis to indicate participant perceived impairment to overall quality of life and disturbance to daily physical activity.

\section{Statistical analysis}

Variables were presented as mean values \pm standard deviation for parametric data or as median (interquartile range - IQR) for nonparametric data. Comparisons between the groups were made using one-way ANOVA or Kruskal Wallis post hoc testing. For categorical variables, chi-square or Fisher's exact test were performed. As there was no significant difference in BMI between groups, ex-smokers with and without COPD were combined for partial correlation analysis. Association between body composition, systemic inflammation and lung function and physical activity was assessed using partial correlation analysis adjusted for age, gender, height, weight and smoking history. Nonparametric variables were transformed. The effects of body composition and systemic inflammation on lung function were further explored using multiple linear regression models. Body composition variables; total body LMM, FM and BMC, systemic inflammation variables; IL-6, CRP and leptin; identified as significant using partial correlation analysis were included as independent variables in the models. The models were adjusted for age, gender, height, weight and smoking history. The robust variance estimator was applied to nonparametric variables to account for the outcome being skewed. P values $<0.05$ were considered statistically significant. Statistical analysis was undertaken using Stata version 11 (StataCorp LP, College Station TX, USA).

\section{Sample size}

With a sample size of 107 participants, and a standard deviation in the never smoker group equal to 9 , there was $80 \%$ power to detect a difference of $7 \mathrm{~kg}$ in total fat between healthy controls (never smokers) and ex-smokers without COPD groups.

\section{Results}

Ex-smokers with COPD were older and had higher smoking pack years than ex-smokers without COPD. Lung function and exercise 
capacity (MSWT) was lower in ex-smokers with COPD than exsmokers without COPD and never smokers (Table 1). Ex-smokers with COPD had significantly higher sputum \%neutrophils and lower \%macrophages than ex-smokers without COPD and never smokers (Table 2).

Demonstrated by a higher SGRQ activity score, ex-smokers with COPD had a greater degree of activity impairment, compared to never smokers and ex-smokers without COPD. Healthy controls (never smokers) had significantly higher vigorous physical activity (METminutes/week) than ex-smokers (Table 3).

In partial correlation analysis ex-smokers, total LMM was positively associated with lung function (post $\mathrm{FEV}_{1}, \mathrm{FVC}$ ) and negatively associated with total SGRQ score. Furthermore, total FM (\%) was negatively associated with lung function (post $\mathrm{FEV}_{1}, \mathrm{FVC}$ ) and positively related to health status (total SGRQ). Total BMC was positively associated with lung function but not health status or exercise capacity (MSWT) (Table 4).

Systemic inflammatory markers (CRP, IL-6 and leptin) had a negative association with lung function in ex-smokers. A positive association was seen between CRP and IL-6 and total SGRQ score (Table 4).

Total LMM was negatively associated with systemic inflammation (CRP, IL-6 and leptin), while BMI and total FM were negatively associated with systemic inflammation (CRP, IL-6 and leptin). No association was seen between total BMC and systemic inflammation (Table 5).

In multiple linear regression models, total LMM, total BMC and age were predictors of lung function (post $\mathrm{FEV}_{1}, \mathrm{FVC}$ and $\mathrm{FEV}_{1} / \mathrm{FVC}$ ) (Table 6). These models explained 77, 78 and 59\% of the variation in $\mathrm{FEV}_{1}, \mathrm{FVC}$ and $\mathrm{FEV}_{1} / \mathrm{FVC}$ respectively. In these models, IL-6, CRP,

Table 1. Subject demographics.

\begin{tabular}{|c|c|c|c|c|}
\hline Characteristic & $\begin{array}{l}\text { Never Smoker } \\
n=16\end{array}$ & $\begin{array}{l}\text { Ex-smoker No COPD } \\
n=68\end{array}$ & $\begin{array}{l}\text { Ex-smoker COPD } \\
\mathrm{n}=\mathbf{2 3}\end{array}$ & $P$ value \\
\hline Age (years) & $62(46-70)$ & $55(39-68)$ & $66(58-76)^{\mathrm{a}}$ & $<0.001$ \\
\hline Gender (M:F) & $5: 11$ & $23: 45$ & $14: 9$ & 0.056 \\
\hline Pack Years & $\mathrm{N} / \mathrm{A}$ & $24(6-68)$ & $35(23-90)^{\mathrm{a}}$ & 0.002 \\
\hline BMI $\left(\mathrm{kg} / \mathbf{m}^{2}\right)$ & $26.3 \pm 4.0$ & $28.8 \pm 5.7$ & $26.5 \pm 4.6$ & 0.082 \\
\hline Post FEV $_{1}$ (L) & $2.82 \pm 0.69$ & $2.96 \pm 0.70$ & $1.63 \pm 0.70^{\mathrm{b}}$ & $<0.001$ \\
\hline Post FEV \% pred & $103(87-110)$ & $98(73-122)$ & $62(42-78)^{b}$ & $<0.001$ \\
\hline Post FVC (L) & $3.64 \pm 0.84$ & $3.78 \pm 0.86$ & $3.15 \pm 1.02^{\mathrm{a}}$ & 0.017 \\
\hline Post FVC\% pred & $105(89-113)$ & $96(72-116)$ & $83(61-106)^{b}$ & 0.001 \\
\hline Post FEV $_{1}$ :FVC & $0.80(0.72-0.81)$ & $0.78(0.70-0.85)$ & $0.50(0.39-0.61)^{\mathrm{b}}$ & $<0.001$ \\
\hline $\begin{array}{l}\text { GOLD } \\
\text { n(I/II/III/IV) }\end{array}$ & $\mathrm{N} / \mathrm{A}$ & $\mathrm{N} / \mathrm{A}$ & $3 / 13 / 5 / 2$ & N/A \\
\hline ICS use n(\%) & $\mathrm{N} / \mathrm{A}$ & $5(7.4)$ & $14(60.9)^{\mathrm{a}}$ & $<0.001$ \\
\hline ICS dose $(\mu \mathrm{g} / \text { day })^{\dagger}$ & N/A & $1000(1000)$ & $1500(1000-2000)$ & 0.186 \\
\hline SABA use n(\%) & N/A & $9(13)$ & $13(57)^{\mathrm{a}}$ & $<0.001$ \\
\hline LA Anticholinergic use n(\%) & $\mathrm{N} / \mathrm{A}$ & $0(0)$ & $13(57)^{a}$ & $<0.001$ \\
\hline SGRQ & $4(0-4)$ & $9(0-45)$ & $27(7-51)^{b}$ & $<0.001$ \\
\hline MSWT (m) & $\begin{array}{l}\mathrm{n}=16 \\
983 \pm 361\end{array}$ & $\begin{array}{l}\mathrm{n}=56 \\
803 \pm 317\end{array}$ & $\begin{array}{l}n=18 \\
674 \pm 334^{c}\end{array}$ & 0.024 \\
\hline
\end{tabular}

Data presented as mean \pm standard deviation, median $(\mathrm{IQR})$ or $\%$ the percentage of subjects with the specified variable. BMI $=$ Body Mass Index, Post FEV $=$ Post bronchodilator forced expiratory volume in one second, Post FVC $=$ Post bronchodilator forced vital capacity, ICS $=$ Inhaled corticosteroids, SABA $=$ Short acting beta agonist, LA $=$ Long acting, SGRQ $=$ St George Respiratory questionnaire, MSWT $=$ Modified shuttle walk test. ${ }^{\mathrm{a}} \mathrm{P}<0.05$ versus ex-smokers no COPD, ${ }^{\text {b }}<0.05$ versus never smokers and ex-smokers no COPD, ${ }^{\mathrm{c}} \mathrm{P}<0.05$ versus never smokers, ${ }^{\dagger}$ Beclomethasone equivalents

Table 2. Systemic and airway inflammation by group.

\begin{tabular}{|c|c|c|c|c|}
\hline & Never Smoker & $\begin{array}{l}\text { Ex-smoker } \\
\text { No COPD }\end{array}$ & $\begin{array}{l}\text { Ex-smoker } \\
\text { COPD }\end{array}$ & P Value \\
\hline \multicolumn{5}{|c|}{ Systemic Inflammation } \\
\hline CRP (mg/L) & $\begin{array}{l}\mathrm{n}=16 \\
1.4(0.5-2.7)\end{array}$ & $\begin{array}{l}\mathrm{n}=68 \\
1.3(0.7-3.1)\end{array}$ & $\begin{array}{l}\mathrm{n}=22 \\
1.7(0.8-7.1)\end{array}$ & 0.320 \\
\hline Il-6 (pg/mL) & $\begin{array}{l}\mathrm{n}=16 \\
1.1(0.7-1.4)\end{array}$ & $\begin{array}{l}\mathrm{n}=65 \\
1.2(0.6-3.9)\end{array}$ & $\begin{array}{l}\mathrm{n}=22 \\
1.7(1.1-5.7)^{\mathrm{b}}\end{array}$ & 0.003 \\
\hline Leptin $(\mathbf{p g} / \mathrm{mL})$ & $\begin{array}{l}n=16 \\
6065(2191-13785)\end{array}$ & $\begin{array}{l}\mathrm{n}=65 \\
8205(793-31704)\end{array}$ & $\begin{array}{l}\mathrm{n}=22 \\
4500(1540-9940)\end{array}$ & 0.203 \\
\hline \multicolumn{5}{|c|}{ Airway Inflammation } \\
\hline TCC $\left(x 10^{6} / \mathrm{mL}\right)^{*}$ & $\begin{array}{l}\mathrm{n}=4 \\
1.6(0.8-3.7)\end{array}$ & $\begin{array}{l}\mathrm{n}=54 \\
3.4(0.9-14.9)\end{array}$ & $\begin{array}{l}\mathrm{n}=16 \\
5.0(2.6-9.1)\end{array}$ & 0.084 \\
\hline \% Eosinophils & $\begin{array}{l}\mathrm{n}=8 \\
1.1(1.0-1.3)\end{array}$ & $\begin{array}{l}\mathrm{n}=59 \\
0.5(0.0-13.0)\end{array}$ & $\begin{array}{l}\mathrm{n}=14 \\
1.2(0.5-5.8)\end{array}$ & 0.056 \\
\hline$\%$ Neutrophils & $29.6 \pm 27.6$ & $35.1 \pm 23.5$ & $58.3 \pm 23.0^{\mathrm{b}}$ & 0.004 \\
\hline$\%$ Macrophages & $53.0(44.8-61.3)$ & $58.3(7.3-94.4)$ & $31.3(16.7-46.5)^{\mathrm{a}}$ & 0.016 \\
\hline eNO (ppb) & $\begin{array}{l}\mathrm{n}=16 \\
22.4(17.7-35.7)\end{array}$ & $\begin{array}{l}\mathrm{n}=68 \\
16.3(7.0-32.8)^{\mathrm{c}}\end{array}$ & $\begin{array}{l}\mathrm{n}=23 \\
19.1(12.6-27.6)\end{array}$ & 0.025 \\
\hline
\end{tabular}

Data presented as mean \pm standard deviation or median $(\mathrm{IQR})$ as appropriate. TCC $=$ Total cell count, eNO $=$ Exhaled nitric oxide, ppb $=$ parts per billion. ${ }^{a} P<0.05$ versus ex-smokers no COPD, ${ }^{\mathrm{b}} P<0.05$ versus never smokers and ex-smokers no COPD, ${ }^{\mathrm{c}} P<0.05$ versus never smokers. 
Table 3. Body composition* and physical activity by group.

\begin{tabular}{|c|c|c|c|c|}
\hline Body Composition & Never Smoker & $\begin{array}{l}\text { Ex-smoker } \\
\text { No COPD }\end{array}$ & $\begin{array}{l}\text { Ex-smoker } \\
\text { COPD }\end{array}$ & $P$ Value \\
\hline & $n=16$ & $n=67$ & $n=18$ & \\
\hline Total LMM (kg) & $46.4 \pm 9.2$ & $47.0 \pm 0.3$ & $46.6 \pm 7.6$ & 0.961 \\
\hline FFM (kg) & $49.1 \pm 9.7$ & $49.9 \pm 10.7$ & $49.1 \pm 7.9$ & 0.936 \\
\hline FFMI $\left(\mathbf{k g} / \mathbf{m}^{2}\right)$ & $17.7 \pm 2.2$ & $17.7 \pm 2.6$ & $17.3 \pm 1.7$ & 0.805 \\
\hline Total FM (kg) & $23.3 \pm 9.0$ & $30.3 \pm 12.8^{c}$ & $25.1 \pm 9.8$ & 0.042 \\
\hline Total BMC (kg) & $2.7 \pm 0.6$ & $2.8 \pm 0.5$ & $2.6 \pm 0.5$ & 0.136 \\
\hline \multicolumn{5}{|l|}{ Physical Activity (PA) } \\
\hline Average steps/day" & $\begin{array}{l}n=15 \\
8243 \pm 2750\end{array}$ & $\begin{array}{l}\mathrm{n}=68 \\
8294 \pm 3969\end{array}$ & $\begin{array}{l}n=16 \\
7342 \pm 2778\end{array}$ & 0.639 \\
\hline SGRQ Activity Score ${ }^{* k *}$ & $\begin{array}{l}\mathrm{n}=16 \\
12(0-12)\end{array}$ & $\begin{array}{l}\mathrm{n}=68 \\
18(0-60)\end{array}$ & $\begin{array}{l}\mathrm{n}=23 \\
47(17-80)\end{array}$ & $<0.001$ \\
\hline Walking MET-min/week ${ }^{k * * *}$ & $\begin{array}{l}\mathrm{n}=10 \\
1287(743-1584)\end{array}$ & $\begin{array}{l}\mathrm{n}=54 \\
1337(0-4752)\end{array}$ & $\begin{array}{l}\mathrm{n}=14 \\
817(462-1040)\end{array}$ & 0.204 \\
\hline Moderate PA MET-min/week ${ }^{k+* k}$ & $\begin{array}{l}\mathrm{n}=11 \\
1560(940-2400)\end{array}$ & $\begin{array}{l}\mathrm{n}=54 \\
1110(0-5960)\end{array}$ & $\begin{array}{l}\mathrm{n}=15 \\
1740(540-5760)\end{array}$ & 0.279 \\
\hline Vigorous PA MET-min/week ${ }^{* * * t}$ & $\begin{array}{l}\mathrm{n}=13 \\
1440(0-2880)^{\mathrm{b}}\end{array}$ & $\begin{array}{l}\mathrm{n}=55 \\
0(0-4800)\end{array}$ & $\begin{array}{l}\mathrm{n}=16 \\
0(0-360)\end{array}$ & 0.038 \\
\hline Total PA Met min/week ${ }^{* * *}$ & $\begin{array}{l}\mathrm{n}=10 \\
4116(3561-5577)\end{array}$ & $\begin{array}{l}\mathrm{n}=51 \\
3258(378-11814)\end{array}$ & $\begin{array}{l}\mathrm{n}=13 \\
4748(2238-6975)\end{array}$ & 0.742 \\
\hline Average Sitting minutes/day ${ }^{k \star * *}$ & $\begin{array}{l}\mathrm{n}=16 \\
285 \pm 137\end{array}$ & $\begin{array}{l}\mathrm{n}=63 \\
322 \pm 156\end{array}$ & $\begin{array}{l}\mathrm{n}=16 \\
272 \pm 158\end{array}$ & 0.410 \\
\hline
\end{tabular}

Data presented as mean \pm standard deviation or median (IQR) as appropriate. ${ }^{*}$ Data derived from DEXA scan. "Average steps/day derived from pedometer daily step count diary, ${ }^{* *} \mathrm{SGRQ} \%$ Activity score derived from activity domain of SGRQ (a higher score indicated a greater perceived limitation to daily physical activity, ${ }^{* * *} \mathrm{Calculated}$ and derived from IPAQ. aP $<0.05$ versus ex-smokers no COPD, ${ }^{b} P<0.05$ versus never smokers and ex-smokers no COPD, ${ }^{\mathrm{c}} P<0.05$ versus never smokers.

Table 4. Relationships between body composition ${ }^{\dagger}$, systemic inflammation (CRP, IL-6 and leptin) and lung function, health related quality of life and walk test in ex-smokers ( $\mathrm{n}=90$ ).

\begin{tabular}{|c|c|c|c|c|c|}
\hline & Post FEV $_{1}$ (L) & Post FVC (L) & Post FEV $/$ FVC & SGRQ (\%) & MSWT (m) \\
\hline Total Lean (kg) & $0.447^{\text {*** }}$ & $0.507^{k * k}$ & 0.114 & $-0.243^{*}$ & 0.097 \\
\hline FFM (kg) & $0.476^{\text {***k}}$ & $0.528^{k * k}$ & 0.144 & $-0.256^{*}$ & 0.096 \\
\hline FFMI $\left(\mathrm{kg} / \mathrm{m}^{2}\right)$ & $0.245^{*}$ & 0.181 & 0.150 & -0.110 & 0.102 \\
\hline Total Fat (\%) & $-0.443^{\text {k\#k }}$ & $-0.518^{* k * k}$ & -0.107 & $0.219^{*}$ & -0.131 \\
\hline BMC (kg) & $0.335^{\text {*t }}$ & $0.234^{*}$ & $0.346^{\text {*t }}$ & -0.163 & -0.083 \\
\hline $\operatorname{CRP}(\mathrm{mg} / \mathrm{L}) \mathrm{n}=90$ & $-0.372^{\text {k*k }}$ & $-0.282^{\text {*k }}$ & $-0.299^{* * *}$ & $0.238^{*}$ & 0.043 \\
\hline IL-6 $(p g / m L) ~ n=87$ & $-0.268^{*}$ & $-0.228^{*}$ & $-0.269^{*}$ & $0.242^{*}$ & 0.045 \\
\hline Leptin $(\mathrm{pg} / \mathrm{mL}) \mathrm{n}=87$ & $-0.378^{\text {k*k }}$ & $-0.413^{k+k *}$ & -0.131 & 0.159 & -0.171 \\
\hline
\end{tabular}

Data presented as partial correlation $r$ values adjusted for age, gender, height, weight and pack years. 'Body composition variables derived from DEXA total body scan. FFM $=$ Fat free mass, FFMI $=$ Fat free mass index $\left(\mathrm{FFM} /\right.$ height $\left.^{2}\right)$, Post FEV $1=$ Post bronchodilator forced expiratory volume in one second, Post FVC $=$ Post bronchodilator forced vital capacity, SGRQ $=$ St George's Respiratory Questionnaire - total $\%$ score, MSWT $=$ Modified shuttle walk test - total distance completed in metres, CRP $=\mathrm{C}$-reactive protein, IL- $6=$ Interleukin-6. ${ }^{*} p \leq 0.05$, ${ }^{* *} p \leq 0.01,{ }^{* * *} p \leq 0.001$.

Table 5. Relationships between systemic inflammation (CRP, IL-6 and leptin) and body composition ${ }^{\dagger}$ in ex-smokers $(\mathrm{n}=90)$.

\begin{tabular}{|c|c|c|c|}
\hline & CRP (mg/L) & IL-6 (pg/mL) & Leptin $(p g / m L)$ \\
\hline BMI $\left(\mathrm{kg} / \mathrm{m}^{2}\right)$ & $0.486^{\text {*k*}}$ & $0.347^{* *}$ & $0.627^{\text {*** }}$ \\
\hline Total Lean (kg) & $-0.367^{k \text { k.k }}$ & $-\mathbf{- 0 . 3 3 3}^{\text {k*k}}$ & $-0.638^{\star \star \star \star *}$ \\
\hline Total Fat (kg) & $0.255^{\star}$ & $0.282^{*}$ & 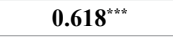 \\
\hline BMC (kg) & -0.012 & 0.135 & -0.128 \\
\hline
\end{tabular}

Data presented as partial correlation $\mathrm{r}$ values adjusted for age, gender, height, weight and pack years. ${ }^{\dagger}$ Body composition variables derived from $\mathrm{DEXA}$ total body scan. CRP $=\mathrm{C}$-reactive protein, IL-6 = Interleukin-6. " $p \leq 0.05,{ }^{* *} p \leq 0.01,{ }^{* * *} p \leq 0.001$

leptin, gender and pack years were not significant predictors of lung function.

\section{Discussion}

This study examined the associations between body composition and lung function, and potential modifiable factors, such as inflammation and physical activity, in ex-smokers. LMM and BMC were found to be positively associated with lung function, while FM was negatively associated with lung function, health status and activity. FM was also positively associated with systemic inflammation (CRP, IL-6 and leptin). Identification of these factors may suggest possible therapeutic targets to improve body composition and hence improve health status in ex-smokers.

\section{Body composition and lung function}

In ex-smokers, LMM and BMC, irrespective of gender or smoking history were positively associated with lung function in both partial correlation and regression models. LMM has previously been shown to be positively associated with lung function, with muscle mass correlating linearly with spirometric values $[21,22]$. In COPD, Poulain et al. reported less severe airflow obstruction in those who were overweight or obese and demonstrated that those individuals also had a greater amount of LMM compared to those of normal weight [6].

Interestingly, while FM was a negative predictor of lung function in partial correlation analysis, in a regression model which included both FM and LMM (Table 6), FM was no longer a significant predictor 
Table 6. Multiple linear regression models describing body composition ${ }^{\dagger}$ and systemic inflammation predictors of lung function $\left(\mathrm{FEV}_{1}, \mathrm{FVC}\right.$ and $\left.\mathrm{FEV}_{1} / \mathrm{FVC}\right)$ in ex-smokers $(\mathrm{n}=90)$.

\begin{tabular}{|c|c|c|c|c|c|c|}
\hline & $\begin{array}{l}\text { Model 1 } \\
\text { Post FEV } 1 \text { (L) }\end{array}$ & & \begin{tabular}{|l|} 
Model 2 \\
Post FVC (L)
\end{tabular} & & $\begin{array}{l}\text { Model 3 } \\
\text { Post FEV } / \text { FVC }\end{array}$ & \\
\hline & $R^{2}=0.768$ & $P \leq 0.001$ & $R^{2}=0.779$ & $P \leq 0.001$ & $R^{2}=0.591$ & $P \leq 0.001$ \\
\hline Predictor Variable & B-Coefficient (95\%CI) & $P$-value & B-Coefficient $(95 \% \mathrm{CI})$ & $P$-value & B-Coefficient $(95 \% \mathrm{CI})$ & $P$-value \\
\hline Total LMM (kg) & $0.208(0.10,0.31)$ & $<0.001$ & $0.165(0.04,0.29)$ & 0.012 & $0.016(0.001,0.03)$ & 0.040 \\
\hline Total FM (kg) & $0.144(0.03,0.26)$ & 0.011 & $0.080(-0.06,0.22)$ & 0.256 & $0.014(-0.01,0.03)$ & 0.190 \\
\hline Total BMC (kg) & $0.871(0.46,1.28)$ & $<0.001$ & $0.704(0.28,1.13)$ & 0.001 & $0.119(0.03,0.20)$ & 0.006 \\
\hline IL-6 (pg/mL) & $-0.054(-0.13,0.02)$ & 0.153 & & & & \\
\hline CRP (mg/L) & & & $-0.002(-0.04,0.03)$ & 0.890 & $-0.004(-0.01,0.01)$ & 0.410 \\
\hline Leptin (pg/mL) & & & $0.00001(-3.60,0.00003)$ & 0.137 & $-4.030(-3.16,2.36)$ & 0.775 \\
\hline Age (years) & $-0.041(-0.05,-0.03)$ & $<0.001$ & $-0.038(-0.05,-0.03)$ & $<0.001$ & $-0.004(-0.01,-0.002)$ & $<0.001$ \\
\hline Gender & $0.492(-0.07,1.05)$ & 0.084 & $0.276(-0.33,0.88)$ & 0.373 & $0.107(-0.01,0.22)$ & 0.071 \\
\hline Height (m) & $-1.070(-2.84,0.70)$ & 0.237 & $1.144(-0.74,3.03)$ & 0.235 & $-0.475(-0.89,-0.06)$ & 0.026 \\
\hline Weight (kg) & $-0.166(-0.27,-0.06)$ & 0.002 & $-0.123(-0.25,0.01)$ & 0.062 & $-0.013(-0.03,0.01)$ & 0.180 \\
\hline Pack Years & $-0.002(-0.01,0.001)$ & 0.239 & $0.003(-0.001,0.01)$ & 0.123 & $-0.001(-0.002,0.000)$ & 0.069 \\
\hline
\end{tabular}

${ }^{\dagger}$ Body composition variables derived from DEXA total body scan. Post $\mathrm{FEV}_{1}=$ post bronchodilator forced expiratory volume in one second, Post $\mathrm{FVC}=$ post bronchodilator forced vital capacity, BMC = Bone Mineral Content, LMM = Lean Muscle Mass, FM = Fat Mass, CRP = C-reactive protein, IL-6 = Interleukin-6.

of lung function. In other populations (elderly, young and in different ethnicities), body fat, has been shown to be negatively associated with lung function [23-25]. This is likely due to both physiological and inflammatory effects [26]. However, our data suggests that in this population of ex-smokers, the influence of LMM as a positive predictor of lung function is relatively more important than the influence of FM.

One possible explanation for the positive association between BMC and lung function, is the role of minerals such as calcium, not only in the maintenance of bone but in muscle function. The depletion of bone calcium stores, reflective of calcium deficiency, has the potential to disrupt the calcium cycle, which controls contraction and relaxation of muscle fibres and therefore may influence the muscle surrounding the lungs [27].

\section{Obesity paradox}

The obesity paradox has been described in COPD and suggests that increased body weight and/or a high BMI are associated with improved lung function and clinical outcomes. Our data suggests that the presence of increased LMM in conjunction with increased BMC, which occurs with obesity [28], may be responsible for the protective role of obesity and improvement in lung function that has previously been observed in COPD. In a longitudinal study of COPD participants, $\mathrm{BMI} \geq 25 \mathrm{~kg} / \mathrm{m}^{2}$ was associated with higher $\mathrm{FEV}_{1}$ and slower decline in $\mathrm{FEV}_{1}$ [29]. However, that study was unable to determine whether this was due to greater FM or LMM. As BMI does not take into account the composition of excess weight, which consists of lean and fat tissue, assessing components of body composition rather than using anthropometry alone may be more useful in assessing ex-smokers at risk of disease.

\section{Systemic inflammation, physical activity and body composition}

Biomarkers of systemic inflammation were negatively associated with lung function, LMM and health related quality of life. Systemic inflammation has previously been associated with reduced exercise capacity and worse health related quality of life [30]. In ex-smokers, leptin was also a strong negative predictor of body LMM and a strong positive predictor of FM, suggesting that strategies targeting leptin reduction may be beneficial. Leptin is released by adipocytes and has a strong correlation with BMI and FM, increasing in proportion to body adiposity. Other pro-inflammatory cytokines such as TNF- $\alpha$, which are elevated in COPD, may also increase leptin levels, while weight loss can decrease leptin levels $[31,32]$. The reduction in systemic inflammation (including leptin) that accompanies weight loss may be beneficial in COPD. Although it is suggested there is an improved prognosis for COPD patients with higher BMIs, weight loss strategies need to be considered with knowledge of body composition, lung function and the presence of other comorbidities.

\section{Limitations}

The inclusion of lung function measures such as total lung capacity and lung volumes would have assisted further in the assessment of FM and LMM on the mechanics of respiration. In this study, ex-smokers with COPD recorded higher than expected overall physical activity levels. This result may reflect: an over-reporting of physical activity; poor sensitivity of a single function pedometer; the ability to walk and/ or run rather than walk alone during the MSWT; not all participants completing all measures; or participants with COPD having greater functional capacity than those routinely seen in a respiratory clinic. The study did not include participants who were underweight or morbidly obese and/or current smokers, who are likely to have the greatest change in body composition, physical activity and inflammation. Nonetheless, using this cross-sectional design, we have identified several important associations between body composition, physical activity, inflammation and lung function in ex-smokers, which warrant further study.

\section{Conclusions}

LMM and BMC are positively associated with lung function in people with or at risk of developing COPD. Perceived physical activity impairment and systemic inflammation were all found to be negatively associated with body composition in ex-smokers. Longitudinal studies observing change in body composition with lung function and strategies such as; pulmonary rehabilitation programmes to improve exercise capacity; and strategies to reduce systemic inflammation including the use of statins, may result in improved body composition and outcomes in ex-smokers with or without COPD.

\section{Acknowledgements}

The authors wish to acknowledge the laboratory staff from Hunter Medical Research Institute (HMRI) Respiratory Research and Hunter Area Pathology Service for assistance with sample processing and analysis and P McElduff and H Powell from HMRI for their statistical advice. 


\section{Funding}

National Health and Medical Research Council, Centre for Clinical Research Excellence.

\section{References}

1. Schols AM, Broekhuizen R, Weling-Scheepers CA, Wouters EF (2005) Body Composition and mortality in chronic obstructive pulmonary disease. Am J Clin Nutr 82: 53-59. [Crossref]

2. Lainscak M, von Haehling S, Doehner W, Sarc I, Jeric T, et al. (2011) Body mass index and prognosis in patients hospitalized with acute exacerbation of chronic obstructive pulmonary disease. J Cachexia Sarcopenia Muscle 2: 81-86. [Crossref]

3. Eisner MD, Blanc PD, Sidney S, Yelin EH, Lathon PV, et al. (2007) Body composition and functional limitation in COPD. Respir Res 8: 7. [Crossref]

4. McDonald VM, Simpson JL, Higgins I, Gibson PG (2011) Multidimensional assessment of older people with asthma and COPD: clinical management and health status. Age Ageing 40: 42-49. [Crossref]

5. Casanova C, Cote C, De Torres JP, Aguirre-Jaime A, Marin JM, et al. (2005) Inspiratory-to-total lung capacity ratio predicts mortality in patients with chronic obstructive pulmonary disease. Am J Respir Crit Care Med 171: 591-597. [Crossref]

6. Poulain M, Doucet M, Drapeau V, Fournier G, Tremblay A, et al. (2008) Metabolic and inflammatory profile in obese patients with chronic obstructive pulmonary disease. Chron Respir Dis 5: 35-41. [Crossref]

7. Graat-Verboom L, Wouters EF, Smeenk FW, van den Borne BE, Lunde R, et al. (2009) Current status of research on osteoporosis in COPD: a systematic review. Eur Respir J34: 209-218. [Crossref]

8. Romme EA, Rutten EP, Smeenk FW, Spruit MA, Menheere PP, et al. (2013) Vitamin D status is associated with bone mineral density and functional exercise capacity in patients with chronic obstructive pulmonary disease. Ann Med 45: 91-96. [Crossref]

9. Richy F, Bousquet J, Ehrlich GE, Meunier PJ, Israel E, et al. (2003) Inhaled corticosteroids effects on bone in asthmatics and COPD patients: a quantitative systematic review. Osteoporos Int 14: 179-190. [Crossref]

10. Mannino DM, Watt G, Hole D, Gillis C, Hart C, et al. (2006) The natural history of chronic obstructive pulmonary disease. Eur Respir J 27: 627-643. [Crossref]

11. Gan WQ, Man SF, Senthilselvan A, Sin DD (2004) Association between chronic obstructive pulmonary disease and systemic inflammation: a systematic review and a meta-analysis. Thorax 59: 574-580. [Crossref]

12. Fernández-Sánchez A, Madrigal-Santillán E, Bautista M, Esquivel-Soto J, MoralesGonzález A, et al. (2011) Inflammation, oxidative stress, and obesity. Int J Mol Sci 12: 3117-3132. [Crossref]

13. Vozoris NT, O'Donnell DE (2012) Prevalence, risk factors, activity limitation and health care utilization of an obese, population-based sample with chronic obstructive pulmonary disease. Can Respir J 19: e18-e24. [Crossref]

14. Bossenbroek L, De Greef MH, Wempe JB, Krijnen WP, Ten Hacken NH (2011) Daily physical activity in patients with chronic obstructive pulmonary disease: a systematic review. COPD 8: 306-319. [Crossref]

15. Masters N, Tutt C (2009) Smoking Pack Years. Available from: www. smokingpackyears.com
16. American Thoracic Society Standardization of spirometry: 1994 update (1995) Am J Respir Crit Care Med 152: 1107-1136. [Crossref]

17. Gibson PG, Simpson JL, Saltos N (2001) Heterogeneity of airway inflammation in persistent asthma : evidence of neutrophilic inflammation and increased sputum interleukin-8. Chest 119: 1329-1336. [Crossref]

18. Campo LA, Chilingaryan G, Berg K, Paradis B, Mazer B (2006) Validity and reliability of the modified shuttle walk test in patients with chronic obstructive pulmonary disease. Arch Phys Med Rehabil 87: 918-922. [Crossref]

19. Singh SJ, Morgan MDL, Scott S, Walters D, Hardman AE (1992) Development of a shuttle walk test of disability in patients with chronic airways obstruction. Thorax 47: 1019-1024. [Crossref]

20. Craig CL, Marshall A, Sjostrom M, Bauman AE, Booth ML, et al. (2003) International Physical Activity Questionnaire: 12-Country Reliability and Validity. Med Sci Sports Exerc 35: 1381-1395. [Crossref]

21. Santana H, Zoico E, Turcato E, Tosoni P, Bissoli L, et al. (2001) Relation between body composition, fat distribution, and lung function in elderly men. Am J Clin Nutr 73: 827-831. [Crossref]

22. Maiolo C, Mohamed EI, Carbonelli MG (2003) Body composition and respiratory function. Acta Diabetol 40 Suppl 1: S32-38. [Crossref]

23. Wannamethee SG, Shaper AG, Whincup PH (2005) Body fat distribution, body composition, and respiratory function in elderly men. Am J Clin Nutr 82: 996-1003. [Crossref]

24. Joshi AR, Singh R, Joshi AR (2008) Correlation of pulmonary function tests with body fat percentage in young individuals. Indian J Physiol Pharmacol 52: 383-388. [Crossref]

25. Jung DH, Shim JY, Ahn HY, Lee HR, Lee JH, et al. (2010) Relationship of body composition and C-reactive protein with pulmonary function. Respir Med 104: 1197 1203. [Crossref]

26. Salome CM, King GG, Berend N (2010) Physiology of obesity and effects on lung function. J Appl Physiol (1985) 108: 206-211. [Crossref]

27. Berchtold MW, Brinkmeier H, Muntener M (2000) Calcium ion in skeletal muscle: its crucial role for muscle function, plasticity, and disease. Physiol Rev 80: 1215-1265. [Crossref]

28. Hannan MT, Felson DT, Dawson-Hughes B, Tucker KL, Cupples LA, et al. (2000) Risk Factors for longitudinal bone loss in elderly men and women: the Framingham Osteoporosis Study. J Bone Miner Res 4: 710-720. [Crossref]

29. Celli BR, Thomas NE, Anderson JA, Ferguson GT, Jenkins CR, et al. Effect of pharmacotherapy on rate of decline of lung function in chronic obstructive pulmonary disease: results from the TORCH study. Am J Respir Crit Care Med 178: 332-338. [Crossref]

30. Broekhuizen R, Wouters EF, Creutzberg EC, Schols AM (2006) Raised CRP levels mark metabolic and functional impairment in advanced COPD. Thorax 61: 17-22. [Crossref]

31. Calikoglu M, Sahin G, Unlu A, Ozturk C, Tamer L, et al. (2004) Leptin and TNF-alpha levels in patients with chronic obstructive pulmonary disease and their relationship to nutritional parameters. Respiration 71: 45-50. [Crossref]

32. Takabatake N, Nakamura H, Abe S et al. (1999) Circulating leptin in patients with chronic obstructive pulmonary disease. Am J Respir Crit Care Med 159: 1215-1219. [Crossref]

Copyright: (C2019 Smart JM. This is an open-access article distributed under the terms of the Creative Commons Attribution License, which permits unrestricted use, distribution, and reproduction in any medium, provided the original author and source are credited. 